\title{
A Behavioral Model for Co-design
}

\author{
He Jifeng * \\ International Institute For Software Technology \\ The United Nations University \\ P.O.Box 3058, Macau \\ jifeng@iist.unu.edu
}

\section{Introduction}

With chip size reaching one million transistors, the complexity of VLSI algorithms - i.e., algorithms implemented as a digital VLSI circuit - is approaching that of software algorithms - i.e., algorithms implemented as code. However, the design methods for circuits that are commonly found in textbooks resemble the low-level machine language programming methods. Selecting individual logical gates and registers in a circuit like selecting individual machine instruction in a program. State transition diagrams are like flowcharts. These methods may have been adequate for small circuit design when they were introduced, but they are not adequate for circuits that perform complicated customer algorithms.

Oftenly we do not build circuits to perform complicated algorithms directly. We build general-purpose processor, and customise them for a particular algorithm by writing a program. For many application, particularly where speed of execution or security is important, a customer-built in circuit is better than the traditional processor-and-software combination. The speed is improved by the absence of the machine language layer and introducing parallelism, whereas security is improved by the impossibility of reprogramming. Moreover, there are space saving compared to a combination of software and processor.

In principle, there is no difference between hardware and software; what can be done with one can be done with the other. For example, an assignment statement $x:=b$, where $x$ is a Boolean variable, can be realised by a clocked circuit, wherein the output port of a combination device which generates the value of expression $b$ is connected to the input port of a register, which is allocated to hold the value of $x$. An incoming clock signal triggers the execution of the circuit which propagates the value of $b$ to the output port of the register. On the other hand, the instruction set of a general-purpose processor can often be described by an interpreter [2, 6].

Out of the previous analysis has come an increasing awareness of the need for behavioural models suited for specifying and reasoning about both programs and digital devices. Contemporary hardware description languages (for example [7. 10 11]) are not sufficient because of the following limitations:

\footnotetext{
* On leave from East China Normal University, Shanghai. This work was partly supported by EPSRC research grant GR/K58708 "Linking theories for computer science"
}

J. Wing, J. Woodcock, J. Davies (Eds.): FM'99, Vol. II, LNCS 1709, pp. 14201438 1999.

(C) Springer-Verlag Berlin Heidelberg 1999 
1. Most such tools are intended much more for simulation than for mathematically sound reasoning.

2. Difficulties arise in developing circuit specifications that may refer to different levels of behavioral abstraction.

3. Existing formal frameworks for such languages are in general too restrictive to deal with the inherent parallelism of digital circuits.

An extended linear-time temporal logic based on intervals was developed in [4, 5, 9] for presenting the kinds of quantitative timing properties and signal transitions that occur in hardware devices. The behaviour of programs and circuits can often be decomposed into successively smaller intervals of activity. State transitions of programs can be characterised by properties relating the initial and final values of variables over interval of times. However in the treatment of hybrid systems where the physical world evolve continuously, this approach seems inappropriate.

We have used the notations of DC (Duration Calculus [12]) to describe hybrid systems. Case studies show that many quantitative timing properties can be handled effectively in DC. Nevertheless, it is not designed to model event-based languages, and lacks the mechanisms to synchronise systems with different time granularity. Section 2 presents a specification language, which is a variant of DC, enriched with a novel parallel operator to integrate systems evolved at various time rate. Its mixed interval structure enables us to model both discrete-time and continuous-time systems. This framework provides a unifying means for presenting the various features of event-based hardware description languages and state-based imperative programming languages.

The main purpose of the mathematical definition of temporal operators is to deduce their interesting properties. These are most elegantly expressed as algebraic laws - equations usually, but sometimes inequations, with implication between formulae rather than equivalence. Section 3 is devoted to the algebraic properties of our specification language. Algebra is well-suited for direct use by engineers in symbolic calculation of parameters and structure of an optimal design. Algebraic proofs by term rewriting are the most promising way in which computers can assist in the process of reliable design.

Section 4 gives a number of tests, known as healthiness conditions, which can be applied to specifications and intermediate designs to maintain their feasibility during the development process. It also explores the mathematical links between theories satisfying individual healthiness conditions, and shows that the set of formulae expressible in each theory is closed under relevant operators.

The VERILOG hardware description language (HDL) [1] is widely used to model the structure and behaviour of digital systems ranging from simple hardware building blocks to complete systems. It has a simulation oriented semantics based on events, i.e., changes to the values of wires and registers. This event semantics can actually model detailed asynchronous behaviour, but is very fine-grained and does not support formal verification. Section 5 shows the utility of our theory in dealing with hardware, and provides an observation-oriented semantics to the core of VERILOG. 
TEMPURA [4, 9] is an imperative language based on interval temporal logic. It has been put forward as a useful tool for reasoning about concurrent programs and hardware. Every TEMPURA statement is a temporal logic formula. TEMPURA is formalised in Section 6 as a sub-theory which satisfies additional healthiness conditions.

\section{Specification Language} reals

Our specification language is based on continuous time represented by

$$
\text { Time }={ }_{d f} \text { Real }
$$

It adopts two types of time intervals: closed continuous interval $[b, e]$ and finite discrete intervals $<t_{0}, \ldots, t_{n}>$. Let $\mathcal{E} \subseteq$ Time, the set $I(\mathcal{E})$ of intervals with the end points of $\mathcal{E}$ is defined by

$$
\begin{aligned}
I(\mathcal{E}) & ={ }_{d f} C I \cup D I \\
C I & ={ }_{d f}\{[b, e] \mid b \leq e \wedge\{b, e\} \subset \mathcal{E}\} \\
D I & ={ }_{d f}\left\{\left\{t_{0}, \ldots, t_{n}\right\} \mid\left\{t_{0}, \ldots, t_{n}\right\} \subseteq \mathcal{E} \wedge\left(t_{0}<\ldots<t_{n}\right)\right\}
\end{aligned}
$$

In the following, we will use $\sigma$ to range over intervals, and $\sigma . b$ and $\sigma . e$ to represent its left and right end points. Adjacent intervals of the same type can be combined into a big one by the catenation operator ${ }^{-}$

$\sigma_{1} \widehat{\widehat{\sigma}} \sigma_{2}={ }_{d f} \sigma_{1} \cup \sigma_{2}$ if $\sigma_{1} . e=\sigma_{2} . b$ and $\left(\left\{\sigma_{1}, \sigma_{2}\right\} \subseteq C I\right.$ or $\left.\left\{\sigma_{1}, \sigma_{2}\right\} \subseteq D I\right)$

We adopt an inclusion-like partial order $\leq$ among intervals

$$
\sigma_{1} \leq \sigma_{2}={ }_{d f}\left(\sigma_{1} \cdot b=\sigma_{2} \cdot b\right) \wedge\left(\sigma_{1} . e=\sigma_{2} \cdot e\right) \wedge\left(\sigma_{1} \subseteq \sigma_{2}\right)
$$

Clearly, this ordering is preserved by the catenation operator.

As a specification mechanism based on interval temporal logic, our language includes

- global variables, which represent constant (i.e., independent of time) and are denoted by lower letters $x, y, \ldots, z$.

- state variables, which stand for real functions over Time, and are denoted by capital letters $X, Y, \ldots, Z$.

- temporal variables, which are identified as real functions on intervals. and denoted by lower letters $u, v, \ldots, w$.

Two specific temporal variables $l$ and $\sharp$ are present to characterise the type of intervals, where the first one stands for the length of interval

$$
l(\sigma)={ }_{d f} \quad \sigma . e-\sigma . b
$$

and the second is used to count the isolated time points of interval

$$
\begin{aligned}
\sharp([b, e]) & =d_{d f} \text { if } b<e \text { then } 0 \text { else } 1 \\
\sharp\left(<t_{0}, \ldots, t_{n}>\right) & ={ }_{d f} n+1
\end{aligned}
$$


The terms of the language can conveniently be defined by induction

(1) global variables are terms.

(2) temporal variables (including $l$ and $\sharp$ ) are terms.

(3) if $X$ is a state variable, then $\overleftarrow{X} \vec{X}$ and $\bigcirc X$ are terms.

(4) if $r_{1}, \ldots, r_{n}$ are terms and $f$ is an n-ary function name, then $f\left(r_{1}, \ldots, r_{n}\right)$ is also a term.

The set of well-formed formulae is generated by the following rules:

(1) if $r_{1}, \ldots, r_{n}$ are terms, and $p$ is an n-ary predicate name, then $p\left(r_{1}, \ldots, r_{n}\right)$ is a well-formed formula

(2) true and false are well-formed formulae.

(3) if $F$ and $G$ are well-formed formulae, so are $\neg F$ and $F \wedge G$ and $\exists h \bullet F$, where $h$ is either a (global or state) variable.

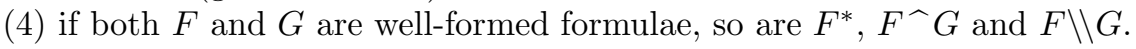

A model $\mathcal{M}$ assigns every variable a constant of the proper type

(1) Temporal variable $v$ is associated with an interval function $\mathcal{M}(v)$.

(2) State variable $X$ is interpreted as a time function $\mathcal{M}(X)$

(3) Global variable $x$ is assigned a real $\mathcal{M}(x)$

(4) Function names $f$ and predicate names $p$ are interpreted as functions $\mathcal{M}(f)$

on Real and relations $\mathcal{M}(p)$ over Real respectively.

Let $h$ be a variable. Two models $\mathcal{M} 1$ and $\mathcal{M} 2$ are called to be $h$ equivalent, denoted by $\mathcal{M} 1 \equiv_{h} \mathcal{M} 2$, if for all variables $v$ different from $x$

$$
\mathcal{M} 1(v)=\mathcal{M} 2(v)
$$

Terms are interpreted over intervals. Given a model $\mathcal{M}$ and an interval $\sigma \in I(\mathcal{E})$ the value of a term $r$ over $\sigma$ is defined by

$$
\begin{aligned}
\mathcal{M}_{\sigma}(x) & { }_{d f} \mathcal{M}(x) \\
\mathcal{M}_{\sigma}(l) & ={ }_{d f} l(\sigma) \\
\mathcal{M}_{\sigma}(\sharp) & { }_{d f} \sharp(\sigma) \\
\mathcal{M}_{\sigma}(v) & ={ }_{d f} \mathcal{M}(v)(\sigma) \\
\mathcal{M}_{\sigma}(\overleftarrow{X}) & { }_{d f} \mathcal{M}(X)(\sigma . b) \\
\mathcal{M}_{\sigma}(\vec{X}) & { }_{d f} \mathcal{M}(X)(\sigma . e) \\
\mathcal{M}_{\sigma}(\bigcirc X) & { }_{d f} \mathcal{M}(X)(\sigma . e) \\
& ={ }_{d f} \mathcal{M}(X)\left(t_{1}\right) \quad \text { if } \sigma \in C I \\
\mathcal{M}_{\sigma}\left(f\left(r_{1}, \ldots, r_{n}\right)\right) & ={ }_{d f} \mathcal{M}(f)\left(\mathcal{M}_{\sigma}\left(r_{1}\right), \ldots, \mathcal{M}_{\sigma}\left(r_{n}\right)\right)
\end{aligned}
$$

Formulae are interpreted as functions from intervals to the Boolean values $\{t t, f f\}$. 


$$
\begin{aligned}
& \mathcal{M}_{\sigma}(\text { true })={ }_{d f} t t \\
& \mathcal{M}_{\sigma}(\text { false })={ }_{d f} f f \\
& \mathcal{M}_{\sigma}\left(p\left(r_{1}, \ldots, r_{n}\right)\right)={ }_{d f} \mathcal{M}_{\sigma}(p)\left(\mathcal{M}_{\sigma}\left(r_{1}\right), \ldots, \mathcal{M}_{\sigma}\left(r_{n}\right)\right) \\
& \mathcal{M}_{\sigma}(\neg F)=_{d f} \neg \mathcal{M}_{\sigma}(F) \\
& \mathcal{M}_{\sigma}(F \wedge G)=_{d f} \mathcal{M}_{\sigma}(F) \wedge \mathcal{M}_{\sigma}(G) \\
& \mathcal{M}_{\sigma}\left(F^{*}\right){ }_{d f} l(\sigma)=0 \vee
\end{aligned}
$$

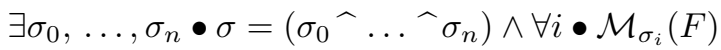

$$
\begin{aligned}
& \mathcal{M}_{\sigma}(\exists h \bullet F)=_{d f} \exists \mathcal{M}^{\prime} \bullet \mathcal{M}_{\sigma}^{\prime}(F) \wedge\left(\mathcal{M} \equiv_{h} \mathcal{M}^{\prime}\right) \\
& \mathcal{M}_{\sigma}\left(F^{\wedge} G\right)={ }_{d f} \exists \sigma_{1}, \sigma_{2} \bullet \sigma=\left(\sigma_{1} \widehat{\sigma_{2}}\right) \wedge \mathcal{M}_{\sigma_{1}}(F) \wedge \mathcal{M}_{\sigma_{2}}(G)
\end{aligned}
$$

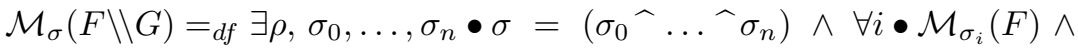

$$
\begin{aligned}
& \rho=<\sigma_{0} . b, \sigma_{1} . b, \ldots, \sigma_{n} . b, \sigma_{n} . e>\wedge \mathcal{M}_{\rho}(G)
\end{aligned}
$$

All the usual logical connectives (disjunction, implication, etc.) and quantifiers can be defined in interval term. For example

$$
\begin{aligned}
& F \vee G={ }_{d f} \neg(\neg F \wedge \neg G) \\
& \forall h \bullet F={ }_{d f} \neg(\exists h \bullet \neg F)
\end{aligned}
$$

The modal operators $\diamond$ and $\square$ can be defined in terms of the chop operator.

(1) The formula $\diamond F$ holds on the interval $\sigma$ if $F$ does so on one of its subintervals.

$$
\diamond F={ }_{d f} \text { true }{ }^{\widehat{\lambda}}\left(F^{\widehat{t r u e}}\right)
$$

$\diamond_{t} F$ holds on $\sigma$ if $F$ holds in its suffix subinterval.

$$
\diamond_{t} F=\operatorname{true}^{\widehat{ }} F
$$

(2) The formula $\square F$ holds if $F$ holds on all its subintervals.

$$
\square F={ }_{d f} \neg \diamond(\neg F)
$$

$\square_{t} F$ holds on $\sigma$ if $F$ holds on all its suffix subintervals.

$$
\square_{t} F={ }_{d f} \neg \diamond_{t}(\neg F)
$$

(3) We use the notation unit to denote the interval of zero length

$$
\text { unit }=_{d f} \quad(l=0)
$$

(4) Let $b$ be a Boolean expression of state variables. Define

$$
F \triangleleft b \triangleright Q={ }_{d f}(F \wedge b) \vee(G \wedge \neg b)
$$

\section{Algebraic Laws}

The great merit of algebra is as a powerful tool for exploring family relationship over a wide range of different theories. For example, study of foundations of interval temporal logic has given denotations to a wide variety of Duration Calculus - Mean Value Calculus of Durations, Extended Duration Calculus, Super-dense 
Duration Calculus, etc. Distinctions are revealed in the structure and content of each kind of time domain and state variable so defined. It is only their algebraic properties that emphasise the family likeness across the range of Duration systems.

\subsection{Chop}

The chop operator ${ }^{-}$is used to model sequential systems. Like its counterpart in ITL, its behaviour is subject to the following familiar laws.

(int-1) (associativity)

$$
F^{\curlywedge}\left(G^{\curlywedge} H\right)=\left(F^{\curlywedge} G\right) \wedge H
$$

(int-2) (unit)

$$
\text { unit }^{\wedge} G=G=G^{\wedge} \text { unit }
$$

(int-3) (disjunctivity)

$$
\begin{aligned}
& F^{\curlywedge}(G 1 \vee G 2)=\left(F^{\curlywedge} G 1\right) \vee\left(F^{\curlywedge} G 2\right) \\
& (F 1 \vee F 2) \frown G=(F 1 \frown G) \vee(F 2 \frown G)
\end{aligned}
$$

(int-4) (conjunctivity) If $L=(l=x)$ or $L=(\sharp=n+1)$ (for $n \geq 0)$, then

$$
\begin{aligned}
& L^{\curlywedge}(G 1 \wedge G 2)=\left(L^{\curlywedge} G 1\right) \wedge\left(L^{\curlywedge} G 2\right) \\
& (F 1 \wedge F 2)^{\curlywedge} L=(F 1 \frown L) \wedge\left(F 2^{\curlywedge} L\right)
\end{aligned}
$$

(int-5) (scope extension) If $h$ is not free in $G$, then

$$
(\exists h \bullet F)^{\curlywedge} G=\exists h \bullet\left(F^{\curlywedge} G\right)
$$

(int-6) (dense time)

$$
(l=r)^{\wedge}(l=s) \Rightarrow(l=r+s)
$$

(int-7) (interval independency) If $F$ does not contain temporal variables, nor temporal propositional letter, then

$$
\begin{aligned}
& \left(F^{\curlywedge} G\right) \Rightarrow F \\
& \left(G^{\curlywedge} F\right) \Rightarrow F
\end{aligned}
$$

(int-8) (zero)

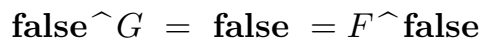

(int-9) (intermediate state) If $p$ is a predicate, then

$$
\left.(F \wedge p(\vec{X}))^{\wedge} G\right)=F^{\wedge}(p(\overleftarrow{X}) \wedge G)
$$

(int-10) (initial and final states) If $p$ is a predicate, then

$$
\begin{aligned}
& (p(\overleftarrow{X}) \wedge F)^{\wedge} Q=p(\overleftarrow{X}) \wedge\left(F^{\wedge} G\right) \\
& F^{\wedge}(G \wedge p(\vec{X}))=(F \wedge G) \wedge p(\vec{X})
\end{aligned}
$$

Adjacent intervals of the same type can be combined into a single one. 
(int-11) (closure of interval catenation)

$$
\begin{aligned}
& (\sharp=0)^{\widehat{\imath}}(\sharp=0)=(\sharp=0) \\
& (\sharp=m+1)^{\wedge}(\sharp=n+1)=(\sharp=m+n+1)
\end{aligned}
$$

(int-12) (validity of catenation)

$$
(\sharp=0)^{\curlywedge}(\sharp \geq 2)=\text { false }(\sharp \geq 2)^{\curlywedge}(\sharp=0)
$$

The length and number of isolated time points of interval are governed by the following laws.

(int-13) (non-empty length)

$$
(\sharp \geq 0)=\text { true }=(l \geq 0)
$$

(int-14) (singleton sets)

$$
(l=0)=(\sharp=1)
$$

\subsection{Parallel}

The definition of $\ \backslash$ is complicated; so it is comforting that it shows many of the algebraic properties of other familiar parallel operators.

$(\backslash \backslash-1)$ (associativity)

$$
(F \backslash \backslash G) \backslash \backslash H=F \backslash \backslash(G \backslash \backslash H)
$$

$(\backslash \backslash-2)$ (time synchronisation)

$F \backslash($ unit $\wedge G)=(F \wedge$ unit $) \backslash G=(F \wedge$ unit $\wedge G)$

$F \backslash \backslash(l>0 \wedge G)=(F \wedge l>0) \backslash \backslash G$

$(\backslash \backslash-3)$ (disjunctivity)

$$
F \backslash \backslash(G 1 \vee G 2)=(F \backslash \backslash G 1) \vee(F \backslash \backslash G 2)
$$

$(\backslash \backslash-4)$ (conjunctivity)

$(F \wedge l=x) \backslash \backslash(G 1 \wedge G 2)=((F \wedge l=x) \backslash \backslash G 1) \wedge\left((F \wedge l=x)^{\wedge} G 2\right)$

$(F 1 \wedge(l=x) \wedge F 2) \backslash \backslash G=((F 1 \wedge l=x) \backslash \backslash G) \wedge((F 2 \wedge l=x) \backslash \backslash G)$

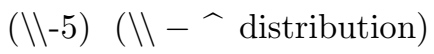

$F \backslash \backslash\left(G^{\curlywedge} H\right)=$

(unit $\backslash \backslash G)^{\wedge}(F \backslash \backslash H) \vee(F \backslash \backslash G)^{\curlywedge}(F \backslash \backslash H) \vee(F \backslash \backslash G)^{\wedge}($ unit $\backslash \backslash H)$

(\\-6) (locality of state variable) If $h$ is not free in $F$, then

$$
F \backslash(\exists h \bullet G)=\exists h \bullet(F \backslash \backslash G)
$$

(\\-7) (monotonicity) If $H_{1} \Rightarrow H_{2}$, then

$$
\left(H_{1} \backslash \backslash G\right) \Rightarrow\left(H_{2} \backslash \backslash G\right)
$$

(\\-8) (exclusiveness)

$\neg($ true $\backslash \backslash(\sharp=0))$ 
$(\backslash \backslash-9)$ (single transition)

$$
F \backslash \backslash(G \wedge \sharp=2)=F \wedge(\text { true } \backslash \backslash(G \wedge \sharp=2))
$$

$(\backslash \backslash-10)$ (void synchronisation)

$$
\text { (true } \backslash \backslash(\sharp=2))=l>0
$$

$(\backslash \backslash-11)(\frown-\vee$ distribution $)$

$$
(\text { unit } \vee F) \backslash \backslash G=(\text { unit } \backslash \backslash G) \vee(F \backslash \backslash G)
$$

$(\backslash \backslash$-12) (interval decomposition independency) If $p$ is a predicate, then

$$
F \backslash \backslash(G \wedge p(\overleftarrow{X}, \vec{X}, l))=(F \backslash \backslash G) \wedge p(\overleftarrow{X}, \vec{X}, l)
$$

(\\-13) (invariant) If $p$ is a predicate, then

$$
(F \wedge p(\overleftarrow{X}, \vec{X})) \backslash \backslash(G \wedge(\sharp \geq 2))=F \backslash \backslash\left(G \wedge \square_{t}(l>0 \Rightarrow p(\overleftarrow{X}, \bigcirc X))\right)
$$

\section{Theorem 3.1}

(1) true $\backslash \backslash$ true $=$ true

(2) false $\backslash \backslash G=$ false $=F \backslash \backslash$ false

(3) $F \backslash \backslash(\sharp \leq 2)=F$

(4) $F \backslash \backslash(\sharp=n+1)=(F \wedge l>0))^{n}, \quad$ for $n \geq 1$

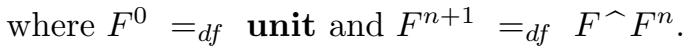

Proof of (1) true $\backslash \backslash$ true $\{($ int -1$)$ and $(\backslash \backslash-3)\}$

$$
\begin{aligned}
& \Leftarrow \text { true } \backslash \backslash(\sharp=1) \vee \text { true } \backslash \backslash(\sharp=2) \\
& =(l=0) \vee(l>0) \\
& =\text { true }
\end{aligned}
$$$$
\{(\backslash-2) \text { and }(\widehat{\wedge}-10)\}
$$$$
\{(\text { int }-1)\}
$$

The conclusion (4) can be proved by induction.

$$
\begin{aligned}
& F \backslash \backslash(\sharp=2) \\
& \{(\backslash \backslash-9) \text { and }(\backslash \backslash-10)\} \\
& =F \wedge(l>0) \\
& F \backslash \backslash(\sharp=n+2) \\
& \{(\text { int }-10)\} \\
& =F \backslash \backslash((\sharp=2) \curlywedge(\sharp=n+1)) \\
& \{(\backslash-5)\} \\
& =(\text { unit } \backslash \backslash(\sharp=2))^{\wedge}(F \backslash \backslash(\sharp=n)) \vee \\
& (F \backslash \backslash \sharp=2)^{\wedge}(F \backslash \backslash \sharp=n) \vee \\
& (F \backslash \backslash(\sharp=2))^{\wedge}(\text { unit } \backslash \backslash(\sharp=n)) \\
& \{(\backslash-2) \text { and }(\text { int }-12)\} \\
& =(F \backslash \Downarrow=2)^{\wedge}(F \backslash \Downarrow=n) \\
& =(F \wedge l>0)^{\wedge}(F \wedge l>0)^{n} \\
& =(F \wedge l>0)^{n+1}
\end{aligned}
$$

\section{Corollary}

If unit $\Rightarrow F$ then $F \backslash \backslash$ true $=F^{*}$ 


\section{Healthiness Conditions}

In this section, we work towards to a more precise characterisation of the class of formulae that are useful in software/hardware design. As usual, we follow the standard practice of mathematics, which is to classify the basic concepts by their important properties. For example, among the functions of real numbers, it is useful to single out those are integrable, or continuous, or rational, or differentiable. A similar classification of the basic concept of a interval formula is essential to our goal of unifying theories of co-design. This section gives a set of healthiness conditions, and shows that the set of healthy formulae is closed under relevant operators. In the later sections we will demonstrate that all actual software/hardware systems satisfy all the the stated healthiness conditions (and more).

\subsection{Monotonicity}

Definition 4.1 (Monotonic formulae)

As a predicate of intervals, a formula $F$ is monotonic if it respects the ordering relation $\leq$ over $I$, i.e., for any model $\mathcal{M}$ and any intervals $\sigma_{1}$ and $\sigma_{2}$

$$
\left(\sigma_{1} \leq \sigma_{2}\right) \Rightarrow\left(\mathcal{M}_{\sigma_{1}}(F) \Rightarrow \mathcal{M}_{\sigma_{2}}(F)\right)
$$

\section{Examples 4.2}

(1) true and false are monotonic.

(2) Let $X$ be a state variable and $p$ a predicate letter, then $p(l, \overleftarrow{X}, \vec{X})$ is monotonic.

(3) The formula $\sharp \leq 1$ is monotonic, but $\sharp \leq n$ is not monotonic for $n \geq 2$.

(4) Let $X$ be a state variable, then

$$
\mathbf{s t b}(X)={ }_{d f} \exists x \bullet \square_{t}(\overleftarrow{X}=x)
$$

is not monotonic.

Define

$$
\Phi_{1}(F)={ }_{d f} \quad F \vee(\text { true } \backslash \backslash F)
$$

Theorem 4.3 (Fixed point representation)

$F$ is monotonic iff $F=\Phi_{1}(F)$

Corollary Monotonic formulae form a complete lattice.

Proof From the monotonicity of $\Phi_{1}$ and Tarski's fixed point theorem.

\section{Theorem 4.4}

$F$ is monotonic iff it lies in the image set of $\Phi_{1}$.

Proof We are going to show that $\Phi_{1}$ is idempotent. 


$$
\begin{aligned}
& \Phi_{1}^{2}(G) \\
= & \Phi_{1}(G) \vee\left(\text { true } \backslash \backslash \Phi_{1}(G)\right) \\
= & \Phi_{1}(G) \vee(\text { true } \backslash \backslash G) \vee((\text { true } \backslash \backslash \text { true }) \backslash \backslash G) \\
= & \Phi_{1}(G) \vee(\text { true } \backslash \backslash G) \\
= & \Phi_{1}(G)
\end{aligned}
$$

$\left\{\right.$ Def of $\left.\Phi_{1}\right\}$

$\left\{\right.$ Def of $\left.\Phi_{1}\right\}$

\section{Theorem 4.5}

(1) $\Phi_{1}(F \vee G)=\Phi_{1}(F) \vee \Phi_{1}(G)$

(2) $\Phi_{1}(F \wedge G) \Rightarrow \Phi_{1}(F) \wedge \Phi_{1}(G)$

(3) $\Phi_{1}(\exists h \bullet F)=\exists h \bullet \Phi_{1}(F)$

(4) $\Phi_{1}\left(F^{\curlywedge} G\right) \Rightarrow \Phi_{1}(F)^{\curlywedge} \Phi_{1}(G)$

(5) $\Phi_{1}\left(F^{*}\right) \Rightarrow\left(\Phi_{1}(F)\right)^{*}$

(6) $\Phi_{1}(F \backslash \backslash G) \Rightarrow\left(\Phi_{1}(F) \backslash \backslash \Phi_{1}(G)\right)$

(7) $\Phi_{1}(F \triangleleft b(\overleftarrow{X}) \triangleright G)=\Phi_{1}(F) \triangleleft b(\overleftarrow{X}) \triangleright \Phi_{1}(G)$

Proof of (3) $\Phi_{1}\left(F^{\widehat{ }} Q\right)$

$$
\begin{array}{rlr}
= & \left(F^{\wedge} G\right) \vee((\text { unit } \backslash \backslash F) \wedge(\text { true } \backslash \backslash G)) \vee & \\
& ((\text { true } \backslash \backslash F) \wedge(\text { true } \backslash \backslash Q)) \vee(\text { true } \backslash \backslash F) \wedge(\text { unit } \backslash \backslash G) & \{(\backslash \backslash-7)\} \\
= & \left(F^{\wedge} G\right) \vee(\text { true } \backslash \backslash F) \wedge(\text { true } \backslash \backslash G) & \{(\text { int }-4)\} \\
\Rightarrow & (F \vee(\text { true } \backslash \backslash F))^{\wedge}(G \vee(\text { true } \backslash \backslash G)) & \left\{\text { Def of } \Phi_{1}\right\} \\
= & \Phi_{1}(P)^{\wedge} \Phi_{1}(Q)
\end{array}
$$

Theorem 4.6 (Closure of monotonic formulae)

If $F$ and $G$ are monotonic, so are $F \vee G, F \wedge G, F^{\curlywedge} G, F^{*}, F \backslash \backslash G, F \triangleleft b(\overleftarrow{X}) \triangleright G$, and $\exists h \bullet F$.

Proof Assume that both $F$ and $G$ are monotonic.

$$
\begin{array}{rlr} 
& F^{\curlywedge} G & \left\{\left(X \Rightarrow \Phi_{1}(X)\right)\right\} \\
\Rightarrow & \Phi_{1}\left(F^{\curlywedge} G\right) & \{\text { Theorem } 4.5(3)\} \\
\Rightarrow & \Phi_{1}(F)^{\curlywedge} \Phi_{1}(G) & \left\{F=\Phi_{1}(F) \text { and } G=\Phi_{1}(G)\right\} \\
= & F^{\curlywedge} G &
\end{array}
$$

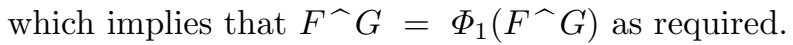

\subsection{Approachable}

Definition 4.7 (DI approachable formulae)

If the behaviour of $F$ over continuous intervals is determined by its behaviour over discrete intervals, then it is said to be $D I$ approachable, i.e.,

$$
\forall \mathcal{M}, \forall \sigma \bullet\left(\sigma \in C I \Rightarrow\left(\mathcal{M}_{\sigma}(F)=\bigvee_{\rho \in D I \wedge \rho \leq \sigma} \mathcal{M}_{\rho}(F)\right)\right)
$$




\section{Examples 4.8}

(1) true, false, unit and $l>0$ are all $D I$ approachable.

(2) $\diamond(X=1)$ is $D I$ approachable.

(3) $\sharp=0$ is monotonic, but not $D I$ approachable.

(4) $\sharp \leq 2$ is $D I$ approachable, but not monotonic.

(5) $\operatorname{stb}(X)$ is neither $D I$ approachable nor monotonic.

Define

$$
\Phi_{2}(F)=_{d f}(\sharp \leq 2) \backslash \backslash F
$$

\section{Theorem 4.9}

$F$ is $D I$ approachable iff $F=\Phi_{2}(F)$

Corollary $D I$ approachable formulae form a complete lattice.

\section{Theorem 4.10}

$F$ is $D I$ approachable iff it lies in the image set of $\Phi_{2}$.

$$
\begin{array}{rrr}
\text { Proof } & (\sharp \leq 2) \backslash \backslash(\sharp \leq 2) & \{(\backslash \backslash-8) \text { and }(\backslash \backslash-3)\} \\
= & (\sharp \leq 2) \backslash \backslash(\sharp=1) \vee(\sharp \leq 2) \backslash \backslash(\sharp=2) & \{(\backslash \backslash-2),(\backslash \backslash-9) \text { and }(\backslash \backslash-10)\} \\
= & (\sharp \leq 2) \wedge(\sharp=1) \vee(\sharp \leq 2) \wedge(l>0) & \\
=\# \leq 2 &
\end{array}
$$

which together $(\backslash \backslash-2)$ implies that $\Phi_{2}$ is idempotent.

\section{Theorem 4.11}

(1) $\Phi_{2}(F \vee G)=\Phi_{2}(F) \vee \Phi_{2}(G)$

(2) $\Phi_{2}(F \wedge G)=\Phi_{2}(F) \wedge \Phi_{2}(G)$

(3) $\Phi_{2}\left(F^{\wedge} G\right)=\Phi_{2}(F)^{\wedge} \Phi_{2}(G)$

(4) $\Phi_{2}(\exists h \bullet F)=\exists h \bullet \Phi_{2}(F)$

(5) $\Phi_{2}\left(F^{*}\right)=\left(\Phi_{2}(F)\right)^{*}$

(6) $\Phi_{2}(F \backslash \backslash G)=\Phi_{2}(F) \backslash \backslash G$

(7) $\Phi_{2}(F \triangleleft b(\overleftarrow{X}) \triangleright G)=\Phi_{2}(F) \triangleleft b(\overleftarrow{X}) \triangleright \Phi_{2}(G)$

Proof $\Phi_{2}\left(F^{\wedge} G\right)$

$$
\begin{aligned}
= & (\text { unit } \backslash \backslash F)^{\wedge} \Phi_{2}(G) \vee \Phi_{2}(F)^{\wedge} \Phi_{2}(G) \vee \\
& \Phi_{2}(F)^{\wedge}(\text { unit } \backslash \backslash G)
\end{aligned}
$$

$=\Phi_{2}(F \wedge \text { unit })^{\wedge} \Phi_{2}(G) \vee \Phi_{2}(F)^{\wedge} \Phi_{2}(G) \vee$

$$
\Phi_{2}(F) \wedge \Phi_{2}(G \wedge \text { unit })
$$

$=\Phi_{2}(F)^{\wedge} \Phi_{2}(G)$

Theorem 4.12 (Closure of $D I$-approachable formulae)

If $F$ and $G$ are $D I$-approachable, so are $F \vee G, F \wedge G, F^{\wedge} G, F^{*}, F \backslash \backslash H$ (for all $H)$ and $\exists h \bullet F$. 


\subsection{Continuity}

\section{Definition 4.13}

A formula $F$ is continuous if for any model and for any time interval $\sigma$

$$
\mathcal{M}_{\sigma}(F)=\bigvee_{\rho \in D I \wedge \rho \leq \sigma} \mathcal{M}_{\rho}(F)
$$

\section{Examples 4.14}

(1) true and false are continuous.

(2) Let $x$ be a global variable. Then the predicate $p(x, l)$ is continuous.

(3) Let $E 1$ and $E 2$ be expressions of state variables. $\vec{E} 1=\overleftarrow{E 2}$ is continuous.

Define

$$
\Phi_{3}(F)=_{d f} \text { true } \backslash \backslash F
$$

\section{Theorem 4.15}

$F$ is continuous iff $F=\Phi_{3}(F)$

Corollary Continuous formulae form a complete lattice.

\section{Theorem 4.16}

$F$ is continuous iff it is monotonic and $D I$ approachable.

$$
\begin{aligned}
& \text { Proof of }(\Rightarrow) \quad \Phi_{2}(F) \\
& \left\{F=\Phi_{3}(F)\right\} \\
& =(\sharp \leq 2) \backslash \backslash(\text { true } \backslash \backslash F) \\
& =((\sharp \leq 2) \backslash \backslash \text { true }) \backslash \backslash F) \\
& =\text { true } \backslash \backslash F \\
& =F \\
& =F \vee \Phi_{3}(F) \\
& \{(\backslash \backslash-1)\} \\
& =\quad F \vee(\text { true } \backslash \backslash F) \\
& =\Phi_{1}(F) \\
& (\Leftarrow) \quad F \\
& =\quad((\sharp \leq 2) \backslash \text { true }) \backslash \backslash F \\
& \left\{(\backslash \backslash-1) \text { and } F=\Phi_{2}(F)=\Phi_{1}(F)\right\} \\
& =\text { true } \backslash \backslash F \\
& =\Phi_{3}(F) \\
& \left\{F=\Phi_{3}(F)\right\} \\
& \left\{F=\Phi_{3}(F)\right\} \\
& \text { \{Def of } \left.\Phi_{3}\right\} \\
& \left\{\text { Def of } \Phi_{1}\right\}
\end{aligned}
$$

\section{Theorem 4.17}

$F$ is continuous iff it lies in the image set of $\Phi_{3}$.

Proof From the fact that true $\backslash \backslash$ true $=$ true.

\section{Theorem 4.18}
(1) $\Phi_{3}(F \vee G)=\Phi_{3}(F) \vee \Phi_{3}(G)$
(2) $\Phi_{3}(F \wedge G) \Rightarrow \Phi_{3}(F) \wedge \Phi_{3}(G)$
(3) $\Phi_{3}\left(F^{\wedge} G\right)=\Phi_{3}(F)^{\wedge} \Phi_{3}(G)$
(4) $\Phi_{3}(\exists h \bullet F)=\exists h \bullet \Phi_{3}(F)$ 
(5) $\Phi_{3}\left(F^{*}\right)=\left(\Phi_{3}(F)\right)^{*}$

(6) $\Phi_{3}(F \backslash \backslash G)=\Phi_{3}(F) \backslash \backslash G$

(7) $\Phi_{2}(F \triangleleft b(\overleftarrow{X}) \triangleright G)=\Phi_{2}(F) \triangleleft b(\overleftarrow{X}) \triangleright \Phi_{2}(G)$

Theorem 4.19 (Closure of continuous formulae)

If $F$ and $G$ are continuous, so are $F \vee G, F \wedge G, F^{\wedge} G, F^{*}, F \backslash \backslash H$ (for all $H$ ) and $\exists h \bullet F$.

\subsection{Temporal Programs}

Let $E$ be an expression of state variables. The formula $\operatorname{stb}(E)$ holds on an interval $\sigma$ if the value of $E$ remains unchanged throughout that interval.

$$
\operatorname{stb}(E)=_{d f} \exists x \bullet \square_{t}(\overleftarrow{E}=x)
$$

The formula $\mathbf{s t b}^{-}(E)$ is true on $\sigma$ if the value of $E$ remains changed except at the end of that interval.

$$
\mathbf{s t b}^{-}(E)=_{d f} \exists x \bullet \square_{t}(l>0 \Rightarrow(\overleftarrow{E}=x))
$$

Let $\underline{E}=<E_{1}, \ldots, E_{n}>$ be a list of expressions. We define

$$
\operatorname{stb}(\underline{E})=_{d f} \operatorname{stb}\left(E_{1}\right) \wedge \ldots \wedge \operatorname{stb}\left(E_{n}\right)
$$

The formula $\mathbf{s t b}^{-}(\underline{E})$ can be defined in a similar way.

$$
\begin{array}{ll}
(\text { stb- }) & \operatorname{stb}(\underline{E}) \wedge \text { unit }=\text { unit }=\mathbf{s t b}^{-}(\underline{E}) \wedge \text { unit } \\
(\text { stb-2) } & \operatorname{stb}(\underline{E})=\mathbf{s t b}^{-}(\underline{E}) \wedge(\underline{E}=\underline{\vec{E}}) \\
(\text { stb-3) } & \operatorname{stb}(\underline{E}) \wedge\left(F^{-} G\right)=(\mathbf{s t b}(\underline{E}) \wedge F)^{\wedge}(\mathbf{s t b}(\underline{E}) \wedge G) \\
(\text { stb-4) } & \operatorname{stb}^{-}(\underline{E}) \backslash \mathbf{s t b}^{-}(\underline{E})=\mathbf{s t b}^{-}(\underline{E}) \\
(\text { stb-5) } & \mathbf{s t b}^{-}(<X, Y, \ldots, Z>)^{*}=\mathbf{s t b}^{-}(X)^{*} \wedge \mathbf{s t b}^{-}(<Y, \ldots, Z>)^{*} \\
(\text { stb-6) } & \mathbf{s t b}^{-}(<X, Y, \ldots, Z>) \backslash \mathbf{s t b}^{-}(\underline{E}(X, Y, \ldots, Z))= \\
& \mathbf{s t b}^{-}(<X, Y, \ldots, Z>)^{*} \wedge \mathbf{s t b}^{-}(\underline{E}(X, Y, \ldots, Z))
\end{array}
$$

Definition 4.20 (Program variable)

A state variable $X$ is a program variable if it is identified as a right continuous step function with finite variability, i.e.

$$
\operatorname{stb}^{-}(X)^{*}=\text { true }
$$

Definition 4.21 (Program)

A formula $F$ is said to be a temporal program of variables $X, Y \ldots, Z$ if

$$
F=\mathbf{s t b}^{-}(<X, Y, \ldots, Z>) \backslash \backslash F
$$

We will use $\underline{V A R}$ to abbreviate the list $\langle X, Y, \ldots, Z\rangle$ of state variables henceforth.

\section{Examples 4.22}

(1) true and false are temporal programs.

(2) From (stb-1) and $(\backslash \backslash-2)$ it follows that unit is a program. 
(3) Let $p$ be a predicate, and $x$ a global variable. From $(\backslash \backslash-12)$ and Corollary of Theorem 3.1 we conclude that $p(l, x)$ is a temporal program.

(4) Let $E 1$ and $E 2$ be expressions of program variables $X, Y, \ldots, Z . \vec{E} 1=\overleftarrow{E 2}$ is a temporal program.

(5) $\mathbf{s t b}^{-}(b)$ and $\mathbf{s t b}(b)$ are temporal programs.

\section{Theorem 4.23}

A monotonic formula $F$ is a temporal program of $X, Y, \ldots, Z$ iff $F$ is continuous.

$$
\begin{aligned}
& \text { Proof of }(\Leftarrow) \quad F \\
& =\text { true } \backslash \backslash F \\
& =\left(\mathbf{s t b}^{-}(\underline{V A R})^{*}\right) \backslash \backslash F \\
& =\left(\mathbf{s t b}^{-}(\underline{V A R}) \backslash \backslash \text { true }\right) \backslash \backslash F \\
& =\mathbf{s t b}^{-}(\underline{V A R}) \backslash(\text { true } \backslash \backslash F) \\
& =\mathbf{s t b}^{-}(\underline{V A R}) \backslash \backslash F \\
& (\Rightarrow \quad F \\
& =\quad F \vee(\text { true } \backslash \backslash F) \\
& =\left(\mathbf{s t b}^{-}(\underline{V A R}) \backslash \backslash F\right) \vee(\text { true } \backslash \backslash F) \\
& =\text { true } \backslash \backslash F
\end{aligned}
$$

$\{F$ is continuous $\}$

\{Corollary of Theorem 3.1\}

$$
\{(\mathbf{s t b}-6)\}
$$

$\{F$ is continuous $\}$

$\{F$ is monotonic $\}$

$\{F$ is a program $\}$

$\{(\backslash \backslash-7)\}$

\section{Theorem 4.24}

$F$ is a temporal program iff there is a formula $G$ such that

$$
F=\mathbf{s t b}^{-}(\underline{V A R}) \backslash \backslash G
$$

$$
\begin{array}{rrr}
\text { Proof } & \text { stb }^{-}(\underline{V A R}) \backslash \backslash\left(\mathbf{s t b}^{-}(\underline{V A R}) \backslash \backslash G\right) & \{(\backslash \backslash-1)\} \\
= & \left(\mathbf{s t b}^{-}(\underline{V A R}) \backslash \backslash \mathbf{s t b}^{-}(\underline{V A R})\right) \backslash \backslash G & \{(\mathbf{s t b}-4)\} \\
=\mathbf{s t b}^{-}(\underline{V A R}) \backslash \backslash G & \square
\end{array}
$$

\section{Theorem 4.25}

(1) Temporal programs form a complete lattice.

(2) If $F$ and $G$ are temporal programs, so are $F \vee G, F^{\wedge} G, F^{*}, F \triangleleft b \triangleright G$ and $\exists h \bullet F$.

Example 4.26 (Temporal assignment)

Let $E 1$ and $E 2$ be the expressions of program variables.

The notation $E 1 \leftarrow E 2$ denotes a temporal assignment, which holds on the interval $\sigma$ if the final value of $E 1$ and the initial value of $E 2$ are the same.

$$
E 1 \leftarrow E 2={ }_{d f} \quad \vec{E} 1=\overleftarrow{E 2}
$$

Example $4.22(3)$ indicates that $E 1 \leftarrow E 2$ is a temporal program. 


\section{Example 4.27 (Assignment)}

Conventional programming language assignments $X:=E$ usually incorporate additional assumptions that are not captured by temporal assignments. Typically, all program variables but $X$ are assumed to remain unchanged, and the update on $X$ occurs at the end of the interval.

$$
X:=E=_{d f}(X \leftarrow E) \wedge\left(\mathbf{s t b}^{-}(X) \wedge \mathbf{s t b}(Y) \wedge \ldots \wedge \operatorname{stb}(Z)\right)
$$

\section{$5 \quad$ VERILOG Timing Controlled Statements}

The VERILOG hardware description language [11] is widely used to model the structure and behaviour of digital systems ranging from simple hardware building blocks to complete systems. Its semantics is based on scheduling of events and the propagation of changes. In this section we are going to examine the VERILOG timing controlled statements and the delayed assignments.

Timing controls are used in VERILOG for scheduling. They are either delay (\#e) or guards. Guards are either level sensitive (wait $(b)$ ) or edge sensitive $(\Delta(b), \Uparrow(b)$ or $\Downarrow(b))$.

Let $P$ be a program, and $e$ an expression. The VERILOG statement \# $\& P$ postpones the execution of $P$ for $e$ time units.

$\# e P={ }_{d f} \quad(l=\overleftarrow{e})^{\wedge} P$

Let $b$ be a Boolean expression of program variables. wait $(b) \& P$ fires the program $P$ whenever the guard $b$ becomes true.

wait $(b) \& P={ }_{d f} \quad\left(\right.$ unit $\left.\triangleleft b \triangleright \mathbf{s t b}^{-}(b)\right) \wedge(P \triangleleft b \triangleright \mathbf{s t b}(b))$

$\Uparrow(b) \& P$ executes the program $P$ whenever the value of $b$ switches from $f f$ to $t$.

$\Uparrow(b) \& P={ }_{d f}\left(\mathbf{s t b}^{-}(b)^{\wedge}(\mathbf{s t b}(b) \triangleleft b \triangleright(\mathbf{w a i t}(b) \& P))\right) \triangleleft b \triangleright(\boldsymbol{w a i t}(b) \& P)$

Its dual, denoted by $\Downarrow(b) \& P$, executes $P$ whenever the value of $b$ goes back to $f f$.

$$
\Downarrow(b) \& P={ }_{d f} \quad \Uparrow(\neg b) \& P
$$

$\Delta(b) \& P$ executes the program $P$ once the Boolean expression $b$ changes its value.

$$
\Delta(b) \& P={ }_{d f} \quad(\text { wait }(\neg b) \& P) \triangleleft b \triangleright(\text { wait }(b) \& P)
$$

The notation $X=\# e E$ denotes a delayed assignment which evaluates the value of $E$, and then assigns it to $X$ after $e$ time units delay.

$$
X=\# e E=_{d f} \exists c \bullet(\overleftarrow{E}=c) \wedge(\# e \&(X \leftarrow c))
$$

The delayed assignment $X=\operatorname{wait}(b) E$ evaluates the value of $E$ first, and assigns it to $X$ whenever $b$ becomes true.

$$
(X=\text { wait } b E)=_{d f} \exists c \bullet(\overleftarrow{E}=c) \wedge(\text { wait }(b) \&(X \leftarrow c))
$$

From Theorem 4.25 it follows that all timing controlled statements and the delayed assignments are temporal programs. 


\section{Clock}

In this section, we choose natural numbers as the end points of the intervals:

$$
\mathcal{E}={ }_{d f} N a t
$$

As a result, every interval has integer length

$$
\exists n \in N a t \bullet(l=n)=\text { true }
$$

and $(\mathbf{s t b}-4)$ can be strengthen to

$(\mathbf{s t b}-4 \mathrm{a}) \quad\left(\mathbf{s t b}^{-}(\underline{E}) \wedge l \leq 1\right) \backslash \backslash \mathbf{s t b}^{-}(\underline{E})=\mathbf{s t b}^{-}(\underline{E})$

\section{Definition 6.1 (Register)}

A state variable $X$ is a register variable if its value changes only at the end points.

$$
\operatorname{stb}^{-}(X)^{*}=\text { true }
$$

Definition 6.2 (Fullness)

A time interval $\sigma$ is full if it contains all the end points of $\mathcal{E}$ lying between $\sigma . b$ and $\sigma . e$, i.e.

$$
\{n \mid n \in \mathcal{E} \wedge(\sigma . b \leq n \leq \sigma . e)\} \subseteq \sigma
$$

All the intervals in $C I$ are full. The discrete interval $<0,1,2\rangle$ is full, but $<0,2>$ is not.

Define

$$
\text { full }=d_{d f}(\sharp=0) \vee(\sharp=l+1)
$$

It is obvious that full holds on $\sigma$ iff $\sigma$ is full. Furthermore, if $\sigma$ is full, so are its subintervals.

$$
\begin{aligned}
& \text { (full-1) } \square \text { full = full } \\
& \text { (full-2) } \square_{t} \text { full }=\text { full }
\end{aligned}
$$

\section{Theorem 6.3}

If $\underline{V A R}={ }_{d f}<X, Y, \ldots, Z>$ is a list of register variables then

$$
\left(\mathbf{s t b}^{-}(\underline{V A R}) \wedge(l \leq 1)\right)^{*}=\text { full }
$$

Definition 6.4 (Clocked program)

$F$ is a clocked program of register variables of $\underline{V A R}$ if

$$
F=\left(\mathbf{s t b}^{-}(\underline{V A R}) \wedge l \leq 1\right) \backslash \backslash F
$$

Theorem 6.5 (Additional healthiness condition)

$F$ is a clocked program iff $F$ is a temporal program satisfying $F=F \wedge$ full

$$
\begin{aligned}
\text { Proof of }(\Rightarrow) & \mathbf{s t b}^{-}(\underline{V A R}) \backslash \backslash F \\
= & \left(\mathbf{s t b}^{-}(\underline{V A R}) \backslash\left(\mathbf{s t b}^{-}(\underline{V A R}) \wedge(l \leq 1)\right)\right) \backslash \backslash F \\
= & \left(\left(\mathbf{s t b}^{-}(\underline{V A R}) \backslash \backslash \mathbf{s t b}^{-}(\underline{V A R})\right) \wedge(l \leq 1)\right) \backslash \backslash F \\
& =\left(\mathbf{s t b}^{-}(\underline{V A R}) \wedge(l \leq 1)\right) \backslash F \\
& =F
\end{aligned}
$$


which implies that $F$ is a temporal program. From $(\backslash \backslash-3)$ and the fact that

$$
F=\left(\mathbf{s t b}^{-}(V A R) \wedge(l \leq 1)\right) \backslash \backslash F
$$

we conclude that $F=F \wedge$ full.

$$
\begin{aligned}
(\Leftarrow) & F \\
= & \left(\mathbf{s t b}^{-}(\underline{V A R}) \backslash \backslash F\right) \\
& =\left(\left(\mathbf{s t b}^{-}(\underline{V A R}) \wedge l \leq 1\right) \backslash \backslash \mathbf{s t b}^{-}(\underline{V A R})\right) \backslash F \\
& =\left(\mathbf{s t b}^{-}(\underline{V A R}) \wedge l \leq 1\right) \backslash F
\end{aligned}
$$

\section{Theorem 6.6}

If $F$ is a clocked program then $F$ is monotonic.

\section{Corollary}

$F$ is a clocked program iff $F$ is continuous and satisfies

$$
F=F \wedge \text { full }
$$

Proof of $(\Rightarrow)$ From Theorems 6.5 and 6.6 it follows that $F$ is a monotonic program satisfying $F=F \wedge$ full. The conclusion that $F$ is also continuous follows directly from Theorem 4.23.

$(\Leftarrow)$ The conclusion follows from Theorems 4.23 and 6.5 .

\section{Theorem 6.7}

(1) Clocked programs form a complete lattice.

(2) If $F$ and $G$ are clocked programs, so are $F^{\wedge} G, F \vee G, F \wedge G,(\neg F \wedge$ full $)$, $\exists x \bullet F, F \triangleleft b(\overleftarrow{X}) \triangleright G, \square_{t} F$ and $\square F$

\section{Examples 6.8}

(1) false and full are clocked programs.

(2) Let $p(\overleftarrow{X}, \vec{X}, l)$ be a predicate. Then $p\left(X, X^{\prime}, l\right) \wedge$ full is a clocked program.

(3) Let $X$ be a register variable. Then $\operatorname{stb}(X) \wedge$ full is a clocked program.

TEMPURA [4, 9, is a computer programming language that is especially good at expression temporal behaviour. The following are several built-in operators for constructing TEMPURA programs.

The notation empty is used to mark termination.

$$
\text { empty }=_{d f} \text { unit }
$$

From the fact that

$$
\left(\mathbf{s t b}^{-}(\underline{V A R}) \wedge(l \leq 1)\right) \backslash \backslash \text { unit }=\mathbf{s t b}^{-}(\underline{V A R}) \wedge \text { unit }=\text { unit }
$$

it follows that empty is a clocked program.

The operator next is used to describe what happen next, where "next" means "after one unit of time".

$$
\text { next } F={ }_{d f}(l=1)^{\wedge} F
$$


From Theorem 6.7 it follows that next preserves clocked programs.

Something is considered to happen always if it happens immediately and then again after each time unit.

$$
\text { always } F={ }_{d f} \square_{t} F
$$

Clearly always also preserves clocked programs.

The formula halt $F$ holds on the interval $\sigma$ iff $F$ holds on the interval $<\sigma . e>$.

$$
\text { halt } F={ }_{d f} \text { always }(\text { full } \wedge(\text { empty } \equiv F))
$$

From Theorem 6.7 it follows that if $F$ is a clocked program so is halt $F$.

The assignment $\operatorname{assign}(A, B)$ asserts that the final value of $A$ is equal to the initial value of $B$.

$$
\operatorname{assign}(A, B)=_{d f}(A \leftarrow B) \wedge \text { full }
$$

From Example 6.8(2) we conclude that $\operatorname{assign}(A, B)$ is a clocked program.

Unit-assignment is an assignment which completes its execution in one time unit.

$$
\operatorname{uassign}(A, B)=_{d f}(l=1) \wedge \operatorname{assign}(A, B)
$$

Both $(l=1)$ and $\operatorname{assign}(A, B)$ are clocked programs, so is their conjunction uassign $(A, B)$.

Another kind of assignment that occurs frequently is initialisation. It can be achieved in zero-time.

$$
\operatorname{iassign}(A, B)=_{d f} \quad e m p t y \wedge \operatorname{assign}(A, B)
$$

From Example 6.8(2) it follows that the initialisation is a clocked program.

TEMPERA statement $\operatorname{gets}(A, B)$ is used to express the repeated assignment.

$$
\left.\operatorname{gets}(A, B)=_{d f} \text { always }(\operatorname{empty} \vee \exists x \bullet x=\overleftarrow{B} \wedge \operatorname{next}(\overleftarrow{A}=x))\right)
$$

From Theorem 6.7 it follows that $\operatorname{gets}(A, B)$ is also a clocked program.

\section{References}

[1] M. Abadi and Z. Manna. Temporal logic programming. Proc. IEEE Symposium on Logic Programming, (1987).

[2] M.R. Barbacci. Instruction Set Processor Specifications (ISPS): The notation and its application. IEEE Trans. Comp. 30: 24-40, (1981).

[3] M. Gordon. Proving a computer correct. Technical Report 42, University of Cambridge Computer Laboratory, (1983).

[4] R.W.S. Hale. Programming in Temporal Logic. Technical Report 173, Computing Laboratory, University of Cambridge, (1980).

[5] J. Halpern, Z. Manna and B. Moszkowski. A hardware semantics based on temporal intervals. In Proc. of 10th Internal Colloquium on Automata, Languages and Programming, 278-291, (1983). 
[6] D. May and R. Shepherd. The transputer implementation of Occam. In Communication Process Architecture, 19-29, (1988).

[7] S. Mazor. A Guide to VHDL. Kluwer Publisher, (1992).

[8] B.C. Moszkowski. A Temporal Logic for multi-level reasoning about hardware. IEEE Computer 18(2): 10-19, (1985).

[9] B.C. Moszkowski. Executing Temporal Logic Programs. Cambridge University Press, Cambridge, (1986).

[10] A.C. Parker and J.J. Wallace. SLOIDE: An I/O hardware description language. IEEE Trans. Comp, 423-439, (1981).

[11] D.E. Thomas and P. Moorby. The VERILOG Hardware Description Language. Kluwer Publisher, (1991).

[12] Zhou Chaochen, C.A.R. Hoare and A.P. Ravn. A calculus of duration. Information Processing Letters 40(5): 269-275, (1991). 